\title{
Numeric Simulation on the Waves from Artificial Anti-gravity upon General Theory of Relativity
}

\author{
Yoshio Matsuki ${ }^{1, *}$, Petro Ivanovich Bidyuk ${ }^{2}$ \\ ${ }^{1}$ National University of Ukraine "Kyiv-Mohyla Academy”, Kyiv 04070, Ukraine \\ ${ }^{2}$ Educational and Scientific Complex "Institute for Applied System Analysis" of the National Technical University of Ukraine "Igor \\ Sikorsky Kyiv Polytechnic Institute”, Kyiv 03056, Ukraine
}

\section{A R T I C L E I N F O \\ Article history: \\ Received: 20 June, 2021 \\ Accepted: 25 September, 2021 \\ Online: 27 September, 2021}

Keywords:

Distorted Time and Space

General Theory of Relativity

Curvature Tensor

\begin{abstract}
A B S T R A C T
This paper reports the algorithm, the input data and the result of the numeric simulation on the flows of the waves emitted from a rotating object that forms the artificial anti-gravity. First, an object with a heavy mass is placed in the 4-dimensional time and space, which is described by a fundamental tensor. Then the first-order derivative of the tensor describes the gravity, and the second-order derivative describes the waves. If the gravity created by the heavy mass is strong enough, time and space become dependent on each other. The input data for the simulation are discrete numbers that surrogate the infinity of the 4-dimensional time-space. The object is assumed to rotate and the tensor equations are solved. Then the coefficients are calculated, which present physical properties of the waves. The result of the simulation shows that the rotating object emits the waves with positive and negative energy intensities, and they have the spin angular momentum that changes its spinning direction upon the selection of the rotation-speed of the object.
\end{abstract}

\section{Introduction}

This research is an extended version of the work presented at the 2020 IEEE $2^{\text {nd }}$ International Conference on System Analysis_Intelligent Computing (SAIC) held in Kyiv, Ukraine, in October 2020 [1] as well as the consequently published paper [2] from the Advances in Science, Technology and Engineering Systems Journal in May 2021. These papers reported that a rotating object would produce anti-gravity, if it held a heavy mass that would distort time and space. The last paper [2] informed of the waves emitted from the rotating object; however, the report of the analysis was forwarded to this new paper because the numeric simulation required a separate research.

An explicit image of the anti-gravity of the rotating object is a flying craft having a disc-shaped body for interstellar travel. Such an object should hold a compact nuclear fusion reactor inside, because the fusion reaction forms the heavier nuclei by fusing the lighter ones such as hydrogen, deuterium and tritium, and this process should distort time and space. However, this is the theory of a black hole [3], and the simulation of the time-space distortion in the nuclear fusion reactor is still the topic of the next research after this paper.

*Corresponding Author: Yoshio Matsuki, matsuki@wdc.org.ua
The waves emitted from the source of the anti-gravity are byproducts, but they can indicate the presence of the source if they have any physical properties such as the energy intensity and the spin angular momentum. The goal of this research is to calculate the energy intensity and the angular momentum of the waves.

In order to describe this phenomenon, the 4-dimensional curvilinear coordinates of the curved space are selected because the heavy object should warp the rectilinear coordinates of the flat space. To start the simulation, the curvature tensor, $\mathrm{R}_{\mu v}$, is given to describe time and space where the object with a heavy mass is placed [3].

$$
\begin{gathered}
R_{\mu \nu}=0 \\
R_{\mu \nu}=\Gamma_{\mu \alpha, v}^{\alpha}-\Gamma_{\mu \nu, \alpha}^{\alpha}-\Gamma_{\mu \nu}^{\alpha} \Gamma_{\alpha \beta}^{\beta}+\Gamma_{\mu \beta}^{\alpha} \Gamma_{\nu \alpha}^{\beta}=0 \\
\Gamma_{\mu \nu \sigma}=\frac{1}{2}\left(g_{\mu \nu, \sigma}+g_{\mu \sigma, v}-g_{\nu \sigma, \mu}\right)
\end{gathered}
$$

This curvature tensor includes the first-order differentials of a fundamental tensor $\left(\mathrm{g}_{\mu v}\right), \mathrm{g}_{\mu v, \sigma}=\partial \mathrm{g}_{\mu v} / \partial \mathrm{x}^{\sigma}$, where $\mathrm{x}^{\sigma}$ is the $\sigma$-th vector (variable) in the given coordinates. Before describing the phenomenon in the curvilinear coordinates, the flat space provides 
a significant introduction to the theory of the waves. Therefore, at first a series of the notations derives the tensor of the waves in an approximate flat space in this introductory section, and then the tensor of the waves is derived in the curved space later in the next section 2.1 .

If the gravitational field is weak, the curvature is also weak and $\mathrm{g}_{\mu v}$ is approximately constant; therefore,

$$
-\Gamma_{\mu \nu}^{\alpha} \Gamma_{\alpha \beta}^{\beta}+\Gamma_{\mu \beta}^{\alpha} \Gamma_{\nu \alpha}^{\beta}=0,
$$

and

$R_{\mu \nu}=\Gamma_{\mu \alpha, v}^{\alpha}-\Gamma_{\mu \nu, \alpha}^{\alpha}=0$

On the other hand,

$$
\begin{aligned}
& R_{\mu \nu \rho \sigma}=\frac{1}{2}\left(g_{\mu \sigma, v \rho}-g_{\nu \sigma, \mu \rho}-g_{\mu \rho, \nu \sigma}+g_{\nu \rho, \mu \sigma}\right) \\
& +\Gamma_{\beta \mu \sigma} \Gamma_{\nu \rho}^{\beta}-\Gamma_{\beta \mu \rho} \Gamma_{\nu \sigma}^{\beta} .
\end{aligned}
$$

By interchanging $\rho$ and $\mu$, and neglecting $\Gamma_{\beta \mu \sigma} \Gamma^{\beta}{ }_{v \rho}-\Gamma_{\beta \mu \rho} \Gamma^{\beta}{ }_{v \sigma}$, we get

$$
\begin{gathered}
R_{\mu \nu}=\mathrm{g}^{\rho \sigma} R_{\mu \nu \rho \sigma} \\
=\mathrm{g}^{\rho \sigma}\left(g_{\rho \sigma, \mu \nu}-g_{\nu \sigma, \mu \rho}-g_{\mu \rho, \nu \sigma}+\mathrm{g}_{\mu \nu, \rho \sigma}\right)=0 .
\end{gathered}
$$

Then

$$
g^{\mu \nu}\left(g_{\mu \nu, \rho \sigma}-g_{\mu \rho, \nu \sigma}-g_{\mu \sigma, \nu \rho}+g_{\rho \sigma, \mu \nu}\right)=0 \text {. }
$$

In general, the moving particle in a scalar field of the potential energy $V$ follows d'Alembert equation,

$$
\nabla \mathrm{V}=\mathrm{g}^{\mu \nu}\left(V_{, \mu \nu}-\Gamma_{\mu \nu}^{\alpha} V_{, \alpha}\right)=0
$$

In order to describe a particle moving in the 4-dimensional time and space, $V$ is replaced by vectors $x^{\lambda}$ of the 4-dimensional coordinates in which $x^{\lambda}, \alpha=g_{\alpha}^{\lambda}$, then d'Alembert equation becomes

$g^{\mu \nu} g_{\alpha, v}^{\lambda}-g^{\mu \nu} g_{\alpha}^{\lambda} \Gamma_{\mu \nu}^{\alpha}=0$

Then

$g^{\mu v} \Gamma_{\mu v}^{\lambda}=0$

Meanwhile,

$\Gamma_{\mu \nu}^{\lambda}=\mathrm{g}^{\lambda} \rho \Gamma_{\rho \mu \nu}=\frac{1}{2} g^{\lambda \rho}\left(g_{\rho \mu, v}+g_{\rho v, \mu}-g_{\mu \nu, \rho}\right)$.

Therefore,

$$
g^{\mu \nu} \Gamma_{\mu \nu}^{\lambda}=\frac{1}{2} g^{\lambda \rho} g^{\mu \nu}\left(g_{\rho \mu, v}+g_{\rho v, \mu}-g_{\mu \nu, \rho}\right)
$$

where

$$
g_{\rho v, \mu}=y_{n, \rho \mu} y_{, v}^{n}+y_{n, v \mu} y_{, \rho}^{n}=y_{n, \rho v} y_{, \mu}^{n}+y_{n, \mu v} y_{, \rho}^{n}=g_{\rho \mu, v},
$$

where $\mu$ and $v$ are in symmetrical relation in the equation, therefore they are exchangeable; and,

$$
y_{, \mu}^{n}=\frac{\partial y^{n}\left(x^{\mu}\right)}{\partial x^{\mu}}
$$

where $\mu=0,1,2,3$, while $x^{\mu}$ are located in $\mathrm{N}$-dimensional physical space of $y^{n}$, where $n=1,2, \ldots . ., \mathrm{N}$.

Therefore,

$$
g^{\mu \nu}\left(g_{\rho \mu, \nu}-\frac{1}{2} g_{\mu \nu, \rho}\right)=0 \text {. }
$$

Then, in order to describe the waves moving in the gravitational field, it is differentiated by $\mathrm{x}^{\sigma}$.

$$
\begin{gathered}
\frac{d}{\mathrm{dx}^{\sigma}} g^{\mu \nu}\left(g_{\rho \mu, \nu}-\frac{1}{2} g_{\mu \nu, \rho}\right) \\
=g_{, \sigma}^{\mu \nu}\left(g_{\rho \mu, \nu}-\frac{1}{2} g_{\mu \nu, \rho}\right)+g^{\mu \nu}\left(g_{\mu \rho, v \sigma}-\frac{1}{2} g_{\mu \nu, \rho \sigma}\right) \\
=g^{\mu \nu}\left(g_{\mu \rho, \nu \sigma}-\frac{1}{2} g_{\mu \nu, \rho \sigma}\right)=0
\end{gathered}
$$

In the equation (17),

$g_{, \sigma}^{\mu \nu}\left(g_{\rho \mu, v}-\frac{1}{2} g_{\mu \nu, \rho}\right)=0$

because $g_{\rho \mu}$ is approximately constant in the weak gravitational field. Therefore,

$g_{\rho \mu, v}=0$

By interchanging $\rho$ and $\sigma$ of (17),

$$
g^{\mu \nu}\left(g_{\mu \sigma, v \rho}-\frac{1}{2} g_{\mu \nu, \sigma \rho}\right)=g^{\mu \nu}\left(g_{\mu \sigma, v \rho}-\frac{1}{2} g_{\mu \nu, \rho \sigma}\right)=0 .
$$

By adding (8), (17) and (20), we get

$g^{\mu \nu} g_{\rho \sigma, \mu \nu}=0$.

It satisfies d'Alembert equation, therefore it describes the waves that move in an empty space where there is only the weak gravitational field. (Note: This equation includes the second-order differentials, $\mathrm{g}_{\mu v, \rho \sigma}=\partial^{2} \mathrm{~g}_{\mu \nu} / \partial \mathrm{x}^{\rho} \partial \mathrm{x}^{\sigma}$.)

The equation (21) describes the waves moving in the weak gravitational field that is approximately a flat 4-dimensional time and space; however, the general theory of relativity predicts the flow of the waves in curvilinear coordinates of the stronger gravitational field. In order to simulate the flow of the waves in the stronger gravitational field, this research includes the 
following terms that have been neglected in the classic reference [3]:

$$
-\Gamma_{\mu \nu}^{\alpha} \Gamma_{\alpha \beta}^{\beta}+\Gamma_{\mu \beta}^{\alpha} \Gamma_{v \alpha}^{\beta} \neq 0
$$

instead of the equation (4),

$$
\Gamma_{\beta \mu \sigma} \Gamma_{v \rho}^{\beta}-\Gamma_{\beta \mu \rho} \Gamma_{v \sigma}^{\beta} \neq 0
$$

for the equation (6), and

$g_{, \sigma}^{\mu \nu}\left(g_{\rho \mu, \nu}-\frac{1}{2} g_{\mu \nu, \rho}\right) \neq 0$

instead of the equation (18).

In this research, the energy intensity and the spin angular momentum of the flow of the waves are simulated as the calculated coefficients of the tensor equation in the spherical polar coordinate system, which is one of the curvilinear coordinate systems. The methodology and the result of the simulation are reported in the following sections.

\section{Methodology}

\subsection{Algorithm}

For the research of the strong gravitational field, $\mathrm{g}_{\mu v}$, is not constant, but given by the following matrix, [3]:

$$
g_{\mu \nu}=\left[\begin{array}{cccc}
g_{00} & 0 & 0 & 0 \\
0 & g_{11} & 0 & 0 \\
0 & 0 & g_{22} & 0 \\
0 & 0 & 0 & g_{33}
\end{array}\right]
$$

$g_{00}=1$

$$
\begin{gathered}
g_{11}=-\frac{2 m}{\mu(\rho-\tau)^{\frac{2}{3}}} \\
g_{22}=-\mu^{2}(\rho-\tau)^{\frac{4}{3}} \\
g_{33}=-\mu^{2}(\rho-\tau)^{\frac{4}{3}} \sin ^{2} \theta
\end{gathered}
$$

And the inverse of $\mathrm{g}_{\mu v}$ is $\mathrm{g}^{\mu v}$. For example,

$g^{11}=-\frac{\mu(\rho-\tau)^{\frac{2}{3}}}{2 m}$

Then $t$ is time, $m$ is the mass of the object, $r$ is the distance from the center of the object, $\theta$ is the angle between $r$ and the rotation axis, $z$, and $\phi$ is the angle of the rotation around the rotation axis in the spherical polar coordinates shown in Figure 1.

$\tau$ is the distorted time and $\rho$ is the distorted distance as defined by the following equations [3].

$\tau=\mathrm{t}+\mathrm{f}(r)$

$\rho=\mathrm{t}+\mathrm{g}(r)$

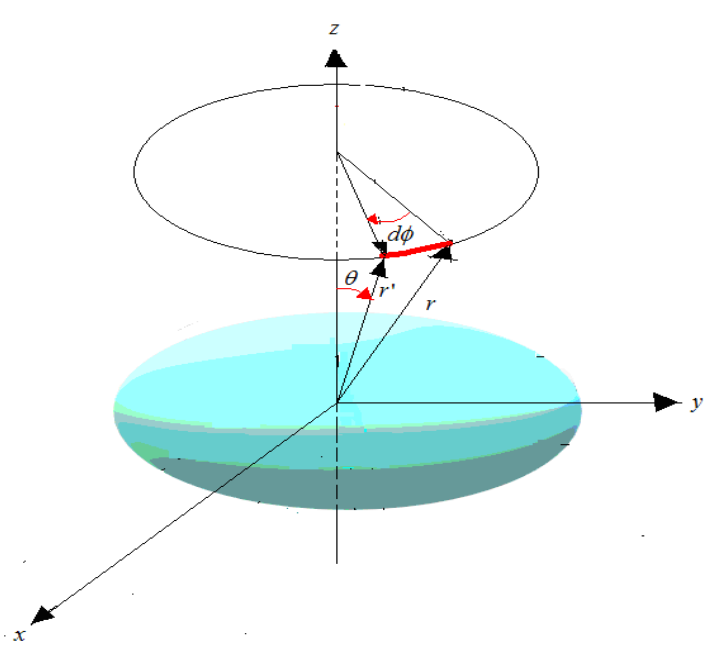

Figure 1: Spherical polar coordinates for the simulation

$\mathrm{f}(\mathrm{r})$ and $\mathrm{g}(\mathrm{r})$ are the functions later defined in the section 2.2. $\mu$ is given by the following equation with the mass, $m$, of the object ([3] p. 35):

$$
\mu=\left(\frac{3}{2} \sqrt{2 m}\right)^{\frac{2}{3}}
$$

The equations below from (34) to (38) show how to derive the equation of the waves in the curved space. It follows the same logic from (7) to (21) in the introductory section above, which derives the equation of the waves in the approximate flat space. But, the equations below include the neglected terms in order to derive the equation in the curved space.

By adding the neglected terms of the equation (23) to the equation (7), we get:

$$
\begin{aligned}
& R_{\mu \nu}=\mathrm{g}^{\rho \sigma} R_{\mu \nu \rho \sigma}=\mathrm{g}^{\rho \sigma}\left(g_{\rho \sigma, \mu \nu}-g_{\nu \sigma, \mu \rho}-g_{\mu \rho, v \sigma}+\mathrm{g}_{\mu \nu, \rho \sigma}\right) \\
& +\mathrm{g}^{\rho \sigma} \Gamma_{\beta \rho \sigma} \Gamma_{\mu \nu}^{\beta}-g^{\rho \sigma} \Gamma_{\beta \mu \rho} \Gamma_{v \sigma}^{\beta} \\
& =\mathrm{g}^{\rho \sigma}\left(g_{\rho \sigma, \mu \nu}-g_{\nu \sigma, \mu \rho}-g_{\mu \rho, v \sigma}+g_{\mu \nu, \rho \sigma}\right) \\
& +g^{\rho \sigma} g_{\beta \beta} \Gamma_{\beta \rho \sigma} \Gamma_{\beta \mu \nu}-g^{\rho \sigma} g_{\beta \beta} \Gamma_{\beta \mu \rho} \Gamma_{\beta v \sigma} \\
& =g^{\rho \sigma}\left(g_{\rho \sigma, \mu \nu}-g_{v \sigma, \mu \rho}-g_{\mu \rho, \nu \sigma}+g_{\mu \nu, \rho \sigma}\right) \\
& +g^{\rho \sigma} g_{\beta \beta} \frac{1}{2}\left(g_{\beta \rho, \sigma}+g_{\beta \sigma, \rho}-g_{\rho \sigma, \beta}\right)\left(g_{\beta \mu, v}+g_{\beta v, \mu}-g_{\mu v, \beta}\right) \\
& -g^{\rho \sigma} g_{\beta \beta} \frac{1}{2}\left(g_{\beta \mu, \rho}+g_{\beta \rho, \mu}-g_{\mu \rho, \beta}\right)\left(g_{\beta v, \sigma}+g_{\beta \sigma, v}-g_{v \sigma, \beta}\right) \\
& =g^{\rho \sigma}\left(g_{\rho \sigma, \mu \nu}-g_{v \sigma, \mu \rho}-g_{\mu \rho, v \sigma}+g_{\mu \nu, \rho \sigma}\right) \\
& +\left(\frac{1}{2} g^{\rho \sigma} g_{\beta \beta} g_{\rho \sigma, \beta} g_{v \sigma, \beta}\right)=0 \text {. }
\end{aligned}
$$

Interchanging $\rho, \mu, \sigma$ and $v$, we get

$$
\begin{aligned}
g^{\mu \nu}\left(g_{\mu \nu, \rho \sigma}\right. & \left.-g_{\mu \rho, v \sigma}-g_{\mu \sigma, \nu \rho}+g_{\rho \sigma, \mu \nu}\right) \\
+\left(\frac{1}{2} g^{\rho \mu} g_{\beta \beta} g_{\rho \mu, \beta} g_{\nu \mu, \beta}\right) & =0 .
\end{aligned}
$$

By adding the neglected terms to the equation (17), we get 


$$
\begin{gathered}
\frac{d}{\mathrm{dx}}\left\{g^{\mu \nu}\left(g_{\rho \mu, \nu}-\frac{1}{2} g_{\mu \nu, \rho}\right)+\frac{1}{2} g^{\rho \mu} g_{\beta \beta} g_{\rho \mu, \beta} g_{\mu \nu, \beta}\right\} \\
=\mathrm{g}_{, \sigma}^{\mu \nu}\left(g_{\rho \mu, \nu}-\frac{1}{2} g_{\mu \nu, \rho}\right)+\mathrm{g}^{\mu \nu}\left(g_{\mu \rho, v \sigma}-\frac{1}{2} g_{\mu \nu, \rho \sigma}\right) \\
+\left(\frac{1}{2} g^{\rho \mu} g_{\beta \beta} g_{\rho \mu, \beta} g_{v \mu, \beta \sigma}\right)+\left(\frac{1}{2} g^{\rho \mu} g_{\beta \beta} g_{\rho \mu, \beta \sigma} g_{v \mu, \beta}\right) \\
+\left(\frac{1}{2} g_{, \sigma}^{\rho \mu} g_{\beta \beta} g_{\rho \mu, \beta} g_{v \mu, \beta}\right) \\
+\left(\frac{1}{2} g^{\rho \mu} g_{\beta \beta, \sigma} g_{\rho \mu, \beta} g_{v \mu, \beta}\right)=0 .
\end{gathered}
$$

Interchanging $\rho$ and $\sigma$ in (36), we get

$$
\begin{array}{r}
g_{, \rho}^{\mu \nu}\left(g_{\sigma \mu, \nu}-\frac{1}{2} g_{\mu \nu, \sigma}\right)+g^{\mu \nu}\left(g_{\mu \sigma, \nu \rho}-\frac{1}{2} g_{\mu \nu, \sigma \rho}\right) \\
+\left(\frac{1}{2} g^{\sigma \mu} g_{\beta \beta} g_{\sigma \mu, \beta} g_{\nu \mu, \beta \rho}\right)+\left(\frac{1}{2} g^{\sigma \mu} g_{\beta \beta} g_{\sigma \mu, \beta \rho} g_{\nu \mu, \beta}\right) \\
+\left(\frac{1}{2} g_{, \rho}^{\sigma \mu} g_{\beta \beta} g_{\sigma \mu, \beta} g_{\nu \mu, \beta}\right)+\left(\frac{1}{2} g^{\sigma \mu} g_{\beta \beta, \rho} g_{\sigma \mu, \beta} g_{\nu \mu, \beta}\right)=0 .
\end{array}
$$

Adding (35), (36) and (37), we get

$$
\begin{aligned}
g^{\mu \nu_{\rho \sigma, \mu \nu}}+g_{, \sigma}^{\mu \nu}\left(g_{\rho \mu, \nu}\right. & \left.-\frac{1}{2} g_{\mu \nu, \rho}\right)+g_{, \rho}^{\mu \nu}\left(g_{\sigma \mu, \nu}-\frac{1}{2} g_{\mu \nu, \sigma}\right) \\
+ & \left(\frac{1}{2} g^{\rho \mu} g_{\beta \beta} g_{\rho \mu, \beta} g_{v \mu, \beta \sigma}\right) \\
+ & \left(\frac{1}{2} g^{\rho \mu} g_{\beta \beta} g_{\rho \mu, \beta \sigma} g_{v \mu, \beta}\right) \\
+ & \left(\frac{1}{2} g_{, \sigma}^{\rho \mu} g_{\beta \beta} g_{\rho \mu, \beta} g_{v \mu, \beta}\right) \\
+ & \left(\frac{1}{2} g^{\rho \mu} g_{\beta \beta, \sigma} g_{\rho \mu, \beta} g_{v \mu, \beta}\right) \\
+ & \left(\frac{1}{2} g^{\sigma \mu} g_{\beta \beta} g_{\sigma \mu, \beta} g_{v \mu, \beta \rho}\right) \\
+ & \left(\frac{1}{2} g^{\sigma \mu} g_{\beta \beta} g_{\sigma \mu, \beta \rho} g_{v \mu, \beta}\right) \\
+\left(\frac{1}{2} g_{, \rho}^{\sigma \mu} g_{\beta \beta} g_{\sigma \mu, \beta} g_{v \mu, \beta}\right) & +\left(\frac{1}{2} g^{\sigma \mu} g_{\beta \beta, \rho} g_{\sigma \mu, \beta} g_{v \mu, \beta}\right)=0 .
\end{aligned}
$$

The equation (38) describes the waves that move in the strong gravitational field. The numbers, $0,1,2$, and 3 , are assigned to each of $\mu, v, \rho, \sigma$ and $\beta$ of the equation (38); then by the rule of tensor, all terms are summed up. Then the mathematical forms of the equation (38) are derived by the functions shown in the equations from (26) to (29). Then according to the equation (25), non-diagonal components such as $\mathrm{g}_{12}$ and $\mathrm{g}_{23}$ vanish; then only diagonal components remain as shown in the equations from (39) to (41). Hereinafter this research focuses on the spatial distribution of the waves but doesn't consider the time. Time is taken into account only by the distorted distance, $\rho$, of the equation (32). Therefore, only three diagonal components of a $3 \times 3$ matrix that represent the spatial distribution of the waves are considered.

$$
\begin{aligned}
& W_{11}=\frac{16}{9(\rho-\tau)^{2}}+\frac{8}{3(\rho-\tau)^{2}}+\frac{64 m}{27 \mu(\rho-\tau)^{\frac{11}{3}}} \\
& -\frac{144 m}{27 \mu(\rho-\tau)^{\frac{13}{3}}}+\frac{8 \mu^{3}}{9 m} \sin ^{2} \theta+\frac{16 \mu^{3}}{18 m} .
\end{aligned}
$$

$$
\begin{gathered}
W_{22}=8 \cos ^{2} \theta-8 \sin ^{2} \theta . \\
W_{33}=\frac{128}{9(\rho-\tau)^{2}}+16 \cot ^{2} \theta .
\end{gathered}
$$

And,

$$
\begin{aligned}
W_{\mu \nu} & =W_{11}+W_{22}+W_{33} \\
& =c_{1} X_{1}+c_{2} X_{2}+\ldots+c_{l} X_{l} .
\end{aligned}
$$

$16 \mu^{3} / 18 \mathrm{~m}$ of the equation (39) is a constant term, and it is not included in the equation (42) for the simulation.

The algorithm to simulate the relative energy intensity of the waves is as follows:

It is assumed that the stress energy tensor, T, reflects the energy intensities of the waves as shown in the equation (43), where $\mathrm{k}$ is a constant.

$$
W_{\mu \nu}=k T
$$

The idea of the stress energy tensor is taken from the classical mechanics [4] (p. 327).

Each of the coefficients, $c_{1}, c_{2} \cdots c_{1}$, relates to a component of the waves such as $1 /(\rho-\tau)^{2}, 1 /(\rho-\tau)^{3 / 11}$, and so on. With the equation (44) we calculate the coefficients, $c_{1}, c_{2} \cdots c_{1}$.

$$
\begin{aligned}
H= & k T-W_{\mu \nu}=k T-\left(c_{1} X_{1}+c_{2} X_{2}+\ldots+c_{l} X_{l}\right) \\
= & k T-\left(c_{1} \frac{1}{(\rho-\tau)^{2}}+c_{2} \frac{1}{(\rho-\tau)^{\frac{11}{3}}}-c_{3} \frac{1}{(\rho-\tau)^{\frac{13}{3}}}\right. \\
& \left.+c_{4} \sin ^{2} \theta+c_{5}\left(\cos ^{2} \theta-\sin ^{2} \theta\right)+c_{6} \cot ^{2} \theta\right)
\end{aligned}
$$

These coefficients make a column vector, c, which holds 1 rows. For calculating c, $X(a n \times l$ matrix) is set as shown in (45), where each of $X_{1}, X_{2}, \cdots X_{l}$ is a vector that holds $n$ rows, and $n$ is also the number of rows of each column of $X$. In this simulation the value of $n$ is 23 as later explained in the section of the input data, and 1 is 6 for the equation (44).

The vectors of the matrix $\mathrm{X}$ should hold the projected image of the energy intensity on the surface of the sphere in the spherical polar coordinates. (Note: The surface of the sphere refers to Figure 14 of [2].

$$
X=\left[\begin{array}{llll}
X_{1} & X_{2} & \ldots & X_{l}
\end{array}\right]
$$

Then $\mathrm{H}$ is set for matrix algebra as shown below.

$$
H=k T-X c
$$

To solve this equation, a constraint is given as shown below, where $X^{\prime}$ is the transposed matrix of $X$.

$$
X^{\prime} H=X^{\prime}(k T-X c)=0
$$

The matrix algebra continues as shown below to calculate the values of $\mathrm{c}$.

$$
\begin{aligned}
& X^{\prime} X c=X^{\prime} k T \\
& c=\left(X^{\prime} X\right)^{-1} X^{\prime} k T
\end{aligned}
$$


here $\left(X^{\prime} X\right)^{-1}$ is an inverse matrix of $X^{\prime} X$.

If the object rotates as shown in Figure 1, its coordinate system is transformed by the transformation matrix D of the Euler's angles [4] as shown below.

$$
D=\left(\begin{array}{ccc}
\cos \phi & \sin \phi & 0 \\
-\sin \phi & \cos \phi & 0 \\
0 & 0 & 1
\end{array}\right)
$$

To simulate the rotation of the object around the axis of $\mathrm{z}$, the matrix, $\mathrm{W}_{\mu v}$, is multiplied by $\mathrm{D}$; then it is transformed as shown below.

$$
\begin{gathered}
D \cdot W_{\mu \nu} \\
=\left[\begin{array}{ccc}
\cos \phi & \sin \phi & 0 \\
-\sin \phi & \cos \phi & 0 \\
0 & 0 & 1
\end{array}\right) \cdot\left[\begin{array}{ccc}
W_{11} & 0 & 0 \\
0 & W_{22} & 0 \\
0 & 0 & W_{33} \\
= & \\
\cos \phi \cdot W_{11} & \sin \phi \cdot W_{22} & 0 \\
-\sin \phi \cdot W_{11} & \cos \phi \cdot W_{22} & 0 \\
0 & 0 & W_{33}
\end{array}\right]
\end{gathered}
$$

To calculate the energy intensity of the waves, the diagonal components are taken from the above transformed matrix, $\mathrm{D} \cdot \mathrm{W}_{\mu \nu}$, then a matrix (52) is set.

$$
\begin{aligned}
& {\left[\begin{array}{ccc}
d W_{11} & 0 & 0 \\
0 & d W_{22} & 0 \\
0 & 0 & W_{33}
\end{array}\right]} \\
& =\left[\begin{array}{ccc}
\cos \phi \cdot W_{11} & 0 & 0 \\
0 & \cos \phi \cdot W_{22} & 0 \\
0 & 0 & W_{33}
\end{array}\right]
\end{aligned}
$$

Then an equation of $\mathrm{H}$ is set as shown below to calculate the coefficients of the diagonal components.

$$
\begin{aligned}
& H=k T-\left(c_{1} \frac{\cos \phi}{(\rho-\tau)^{2}}+c_{2} \frac{\cos \phi}{(\rho-\tau)^{\frac{11}{3}}}-c_{3} \frac{\cos \phi}{(\rho-\tau)^{\frac{13}{3}}}\right. \\
& +c_{4} \cos \phi \sin ^{2} \theta+c_{5} \cos \phi\left(\cos ^{2} \theta-\sin ^{2} \theta\right) \\
& \left.+c_{6} \frac{1}{(\rho-\tau)^{2}}+c_{7} \cot ^{2} \theta\right)
\end{aligned}
$$

Hereinafter the same procedure follows as explained from the equation (44) to (49) to calculate the coefficients.

To calculate the spin angular momentum, a matrix is set as shown in (54), by taking out the anti-symmetrical components of $\mathrm{D} \cdot \mathrm{W}_{\mu \nu}$ from (51).

$$
\left[\begin{array}{ccc}
0 & \sin \phi \cdot W_{22} & 0 \\
-\sin \phi \cdot W_{11} & 0 & 0 \\
0 & 0 & 0
\end{array}\right]
$$

Then a column vector is set as shown below.

$$
\left[\begin{array}{c}
\sin \phi W_{22} \\
-\sin \phi W_{11} \\
0
\end{array}\right]=\left[\begin{array}{c}
W_{22} \cdot d \Omega_{3} \\
-W_{11} \cdot d \Omega_{3} \\
0
\end{array}\right]
$$

With the above column vector, an equation of $\mathrm{H}$ is set as shown below for the calculation of the coefficients.

$$
\begin{gathered}
H=k T-\left\{-c_{1} \frac{-\sin \phi}{(\rho-\tau)^{2}}-c_{2} \frac{-\sin \phi}{(\rho-\tau)^{\frac{11}{3}}}+c_{3} \frac{-\sin \phi}{(\rho-\tau)^{\frac{13}{3}}}\right. \\
\quad-c_{4}(-\sin \phi) \sin ^{2} \theta+c_{5} \sin \phi\left(\cos ^{2} \theta-\sin ^{2} \theta\right)
\end{gathered}
$$

The spin angular momentum is to be projected on the flat surface, which is perpendicular to the curved spherical surface in the spherical polar coordinate system. (Note: The flat surface refers to Figure 15 of [2].

\subsection{Input data}

Figure 2 and Figure 3 show the input data that simulate the distortion of time and space, which we used also for the previous research $[1,2]$. In these figures, $r$ is the distance from the center of the strong gravity; $t$ is the time to travel for the distance; $\tau$ is the distorted time by the equation (31), which expands and shrinks depending on $\mathrm{r}$ and $\mathrm{t}$; and, $\rho$ is the distorted distance by the equation (32), which expands and shrinks depending on $t$ and $r$.

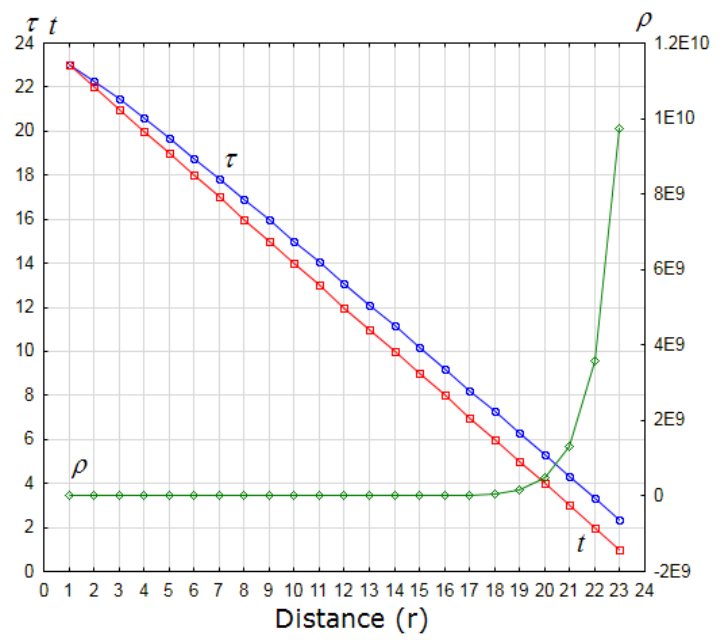

Figure 2: Time and distance from the center of the gravity, Case-1 (non-linear model): $\mathrm{f}(\mathrm{r})=\log \mathrm{r}$ and $\mathrm{g}(\mathrm{r})=\mathrm{e}^{\mathrm{r}}$

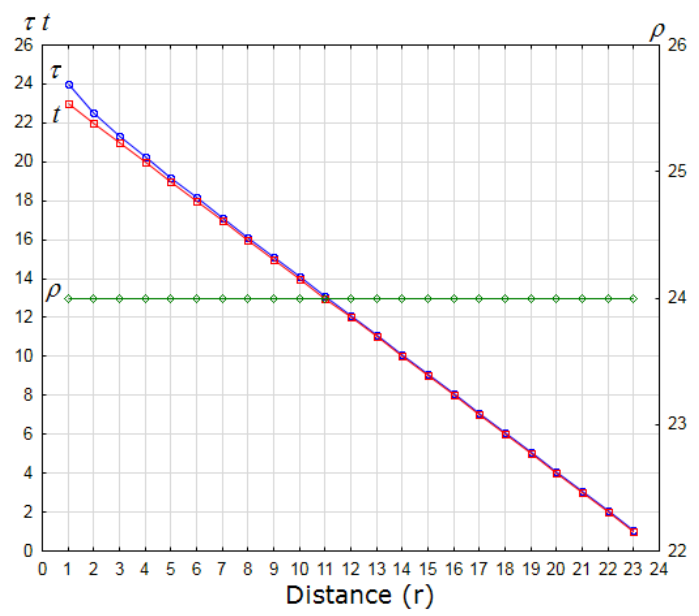

Figure 3: Time and distance from the center of the gravity, Case-2 (linear model): $f(r)=1 / r$ and $g(r)=r$

For the distorted time and space, two models are set: Case-1 (non-linear model) and Case-2 (linear model) as shown in Table 
1 , which assign the functions of $\mathrm{f}(\mathrm{r})$ of the equation (31) and $\mathrm{g}(\mathrm{r})$ of the equation (32).

Table 1: Two models for simulating the distorted time-space

\begin{tabular}{|c|c|c|}
\hline & $\begin{array}{c}\text { Case-1 } \\
\text { (Non-linear model) }\end{array}$ & $\begin{array}{c}\text { Case-2 } \\
\text { (Linear model) }\end{array}$ \\
\hline$f(r)$ & $\log \cdot r$ & $1 / r$ \\
\hline$g(r)$ & $e^{r}$ & $r$ \\
\hline
\end{tabular}

Then the distributions of $\mathrm{r}$ and $\mathrm{t}$ are set to simulate the description of the reference [3], "Any signal, even a light signal, would take an infinite time to cross the boundary of a black hole". In our research the term, a black hole, is replaced by the strong gravity that distorts time and space. However it is not possible to set the infinite value for the numeric simulation of this research that uses a personal computer. Therefore we used 24 discrete finite values as the surrogate that simulates "It takes more time to travel closer to the center of the strong gravity".

The time, $t$, and the distance, $r$, are set as shown in Figure 2 for Case- 1 and Figure 3 for Case-2. For simulating the waves, we assumed that $\theta$ would become smaller in far distance from the object as shown in Figure 4, because the spin of the waves should stabilize the flow as the waves move forward from the center of the gravity; then the angle $\theta$ should be focused and smaller in the far distance.

For simulating the rotation of the object, two modes of the frequencies are set: $\phi_{1}$ (rotation 1) and $\phi_{2}$ (rotation 2) also as shown in Figure 4 . The frequency $\phi_{2}$ is higher than $\phi_{1}$; therefore, rotation 2 represents the faster rotation of the object than rotation 1 . With these settings, $\sin \theta, \cos \theta, \cot \theta, \sin \phi_{1}, \sin \phi_{2}, \cos \phi_{1}$ and $\cos \phi_{2}$ change their values as shown in Figure 5.

The value of the stress energy tensor is set as 1 ; therefore, this simulation doesn't calculate absolute values of the intensities, but relative intensities. The calculated coefficients have dimensions. For example, the coefficient of the first term of the equation (39), $16 /\left\{9(\rho-\tau)^{2}\right\}$, has the dimension of the inverse of $(\rho-\tau)^{2}$ that is the squared dimension of the difference between the distorted distance, $\rho$, and the distorted time, $\tau$.

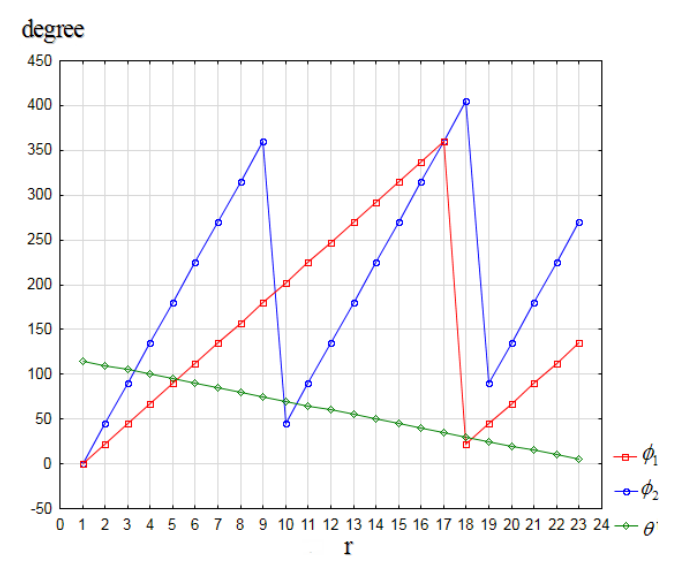

Figure 4: Angles, $\theta, \phi_{1}$ and $\phi_{2}$ for the simulation

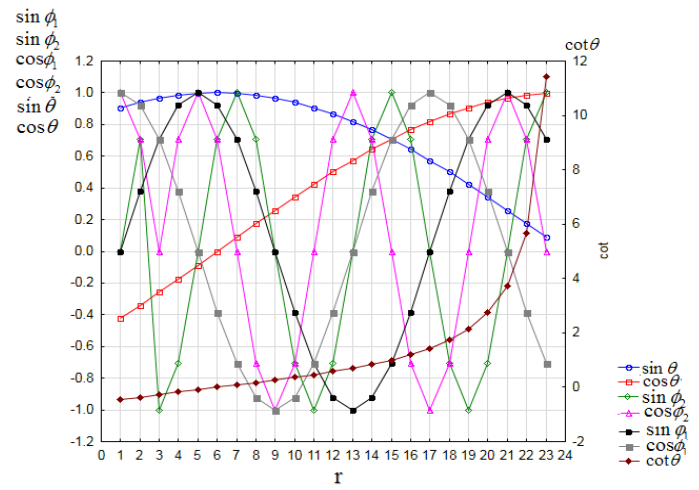

Figure 5: Trigonometric functions, $\sin \theta, \cos \theta, \cot \theta, \sin \phi_{1,}, \sin \phi_{2}, \cos$ $\phi_{1}$ and $\cos \phi_{2}$ for the simulation

\section{3. $\quad$ Result}

\subsection{Energy intensity of the waves}

The calculated energy intensities of the waves are shown in Table 2 for Case-1 (non-linear model) and Table 3 for Case- 2 (linear model). The first and the third columns of these tables are the mathematical forms of the components of the waves, which are taken from the equations from (39) to (41), but each of the constant factors is simplified to unity. For example, $16 /\left\{9(\rho-\tau)^{2}\right\}$ is simplified to $1 /(\rho-\tau)^{2}$ because the factor, $16 / 9$, is not necessary as it is to be included in the coefficient when it is calculated by the equation (49).

The calculated coefficients have either positive or negative values. For example, in Case-1 (non-linear model) the calculated coefficient of $1 /(\rho-\tau)^{2}$ is $4.081 \cdot 10^{-6}$ with no rotation; and, it changes to $-1.465 \cdot 10^{4}$ with rotation 1 , and then $4.178 \cdot 10^{3}$ with rotation 2. It suggests that the energy intensity of this component of the waves changes its sign from negative to positive when the object rotates faster from rotation 1 to rotation 2 .

In order to interpret the calculated results, hereinafter we define that the positive flow holds positive $(+)$ coefficient, and the negative flow holds negative (-) coefficient. For example in the above case of $1 /(\rho-\tau)^{2}$, the wave is a negative flow with rotation 1 ; but, it changes to a positive flow with rotation 2 .

Table 2: Results of the simulation of energy intensity of the waves, Case-1

\begin{tabular}{|c|c|c|c|c|}
\hline $\begin{array}{c}\text { Components } \\
\text { of Waves } \\
\text { (No rotation) }\end{array}$ & $\begin{array}{c}\text { Coefficients } \\
\text { (No } \\
\text { rotation) }\end{array}$ & $\begin{array}{c}\text { Components of } \\
\text { Waves with the } \\
\text { rotation }\end{array}$ & $\begin{array}{l}\text { Coefficients } \\
\text { (Rotation 1) }\end{array}$ & $\begin{array}{c}\text { Coefficients } \\
\text { (Rotation 2) }\end{array}$ \\
\hline $1 /(\rho-\tau)^{2} *$ & $4.081 \cdot 10^{-6}$ & $\cos \phi /(\rho-\tau)^{2}$ & $-1.465 \cdot 10^{4}$ & $4.178 \cdot 10^{3}$ \\
\hline $1 /(\rho-\tau)^{11 / 3}$ & $-1.892 \cdot 10^{-4}$ & $\cos \phi /(\rho-\tau)^{11 / 3}$ & $1.682 \cdot 10^{5}$ & $-2.311 \cdot 10^{5}$ \\
\hline$-1 /(\rho-$ & $-3.270 \cdot 10^{-4}$ & $-\cos \phi /(\rho-\tau)^{13 / 3}$ & $2.836 \cdot 10^{5}$ & $-4.028 \cdot 10^{5}$ \\
\hline$\tau)^{13 / 3}$ & 2.000 & $\cos \phi \sin ^{2} \theta$ & $8.397 \cdot 10^{-2}$ & $3.875 \cdot 10^{-2}$ \\
\hline $\sin ^{2} \theta$ & 1.000 & $\cos \phi\left(\cos ^{2} \theta-\sin ^{2} \theta\right)$ & 1.436 & $-6.610 \cdot 10^{-2}$ \\
\hline $\cos ^{2} \theta-\sin ^{2} \theta$ & - & $1 /(\rho-\tau)^{2} *$ & $1.041 \cdot 10^{4}$ & $3.825 \cdot 10^{2}$ \\
\hline- & $-4.358 \cdot 10^{-10}$ & $\cot ^{2} \theta *$ & $7.534 \cdot 10^{-2}$ & $8.819 \cdot 10^{-2}$ \\
\hline $\cot ^{2} \theta$ & \multicolumn{3}{|r}{} &
\end{tabular}

Notes for Table 2 and 3.

*: This component belongs to both $\mathrm{W}_{11},\left(16 / 9(\rho-\tau)^{2}+8 / 3(\rho-\tau)^{2}\right.$, of the equation (39) and $\mathrm{W}_{33}$, $\left(128 / 9(\rho-\tau)^{2}\right.$, of the equation (41). 
*: These components are on the axis of the rotation and belong to $\mathrm{W}_{33}$; therefore, $\cos \phi$ is not multiplied.

Table 3: Results of the simulation of energy intensity of the waves, Case-2

\begin{tabular}{|c|c|c|c|c|}
\hline $\begin{array}{c}\text { Components } \\
\text { of Waves } \\
\text { (No rotation) }\end{array}$ & $\begin{array}{l}\text { Coefficients } \\
\text { (No } \\
\text { rotation) }\end{array}$ & $\begin{array}{c}\text { Components of } \\
\text { Waves with the } \\
\text { rotation }\end{array}$ & $\begin{array}{l}\text { Coefficients } \\
\text { (Rotation 1) }\end{array}$ & $\begin{array}{l}\text { Coefficients } \\
\text { (Rotation 2) }\end{array}$ \\
\hline $1 /(\rho-\tau)^{2} *$ & $-1.563 \cdot 10^{-7}$ & $\cos \phi /(\rho-\tau)^{2}$ & -17.34 & -63.97 \\
\hline $1 /(\rho-\tau)^{11 / 3}$ & $2.206 \cdot 10^{-6}$ & $\cos \phi /(\rho-\tau)^{11 / 3}$ & $-1.313 \cdot 10^{2}$ & $1.156 \cdot 10^{3}$ \\
\hline$-1 /(\rho-$ & $2.493 \cdot 10^{-6}$ & $-\cos \phi /(\rho-\tau)^{13 / 3}$ & $-1.460 \cdot 10^{2}$ & $1.390 \cdot 10^{3}$ \\
$\tau)^{13 / 3}$ & 2.000 & $\cos \phi \sin ^{2} \theta$ & 0.1066 & $6.768 \cdot 10^{-2}$ \\
\hline $\sin ^{2} \theta$ & 1.000 & $\cos \phi\left(\cos ^{2} \theta-\sin ^{2} \theta\right)$ & 0.4353 & -0.5905 \\
\hline $\cos ^{2} \theta-\sin ^{2} \theta$ & - & $1 /(\rho-\tau)^{2} *$ & 28.03 & 12.58 \\
\hline- & 10 & $\cot ^{2} \theta *$ & $8.474 \cdot 10^{-2}$ & $8.236 \cdot 10^{-3}$ \\
\hline $\cot ^{2} \theta$ & $-4.306 \cdot 10^{-}$ & & & \\
\hline
\end{tabular}

The summations of the values of each of the positive coefficients and the negative coefficients are shown in Table 4 for both Case- 1 and Case-2, and Figure 6 for Case- 1 and Figure 7 for Case-2. In both cases, positive and negative flows of the waves are not visible in the figures when the object doesn't rotate; but, they become visible when the object rotates.

Table 4: Energy intensities of positive and negative flows

\begin{tabular}{|l|c|c|}
\hline & Positive flow & Negative flow \\
\hline No rotation (Case-1) & 3.000 & $-4.358 \cdot 10^{-10}$ \\
\hline Rotation 1 (Case-1) & $4.622 \cdot 10^{5}$ & $-1.465 \cdot 10^{4}$ \\
\hline Rotation 2 (Case-1) & $4.560 \cdot 10^{3}$ & $-6.339 \cdot 10^{5}$ \\
\hline No rotation (Case-2) & 3.000 & $-1.567 \cdot 10^{-7}$ \\
\hline Rotation 1 (Case-2) & $2.865 \cdot 10^{1}$ & $-2.947 \cdot 10^{2}$ \\
\hline Rotation 2 (Case-2) & $2.558 \cdot 10^{3}$ & $-6.456 \cdot 10^{1}$ \\
\hline
\end{tabular}

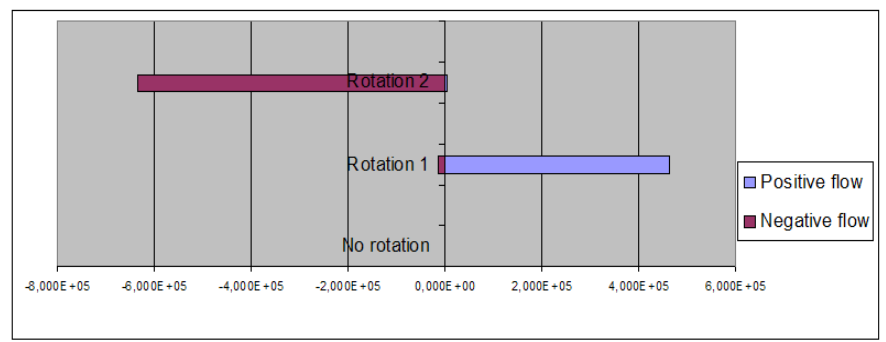

Figure 6: Energy intensity of positive and negative flows: (Case-1)

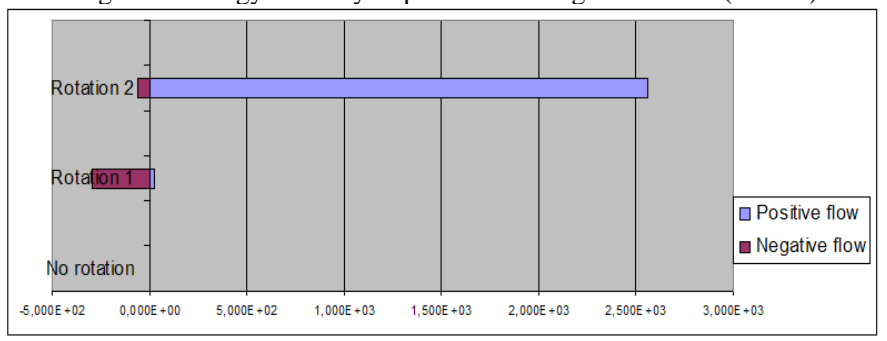

Figure 7: Energy intensity of positive and negative flows: (Case-2)

\subsection{Spin angular momentum}

Table 5 and Table 6 show the calculated relative intensity of the spin angular momentum of the waves, which are projected in the directions of $\sin \phi \cdot \mathrm{W}_{22}$ and $-\sin \phi \cdot \mathrm{W}_{11}$. (Note: $\sin \phi \cdot \mathrm{W}_{22}$ and $\sin \phi \cdot \mathrm{W}_{11}$ are perpendicular to the rotation axis.)

The calculated coefficients of the spin angular momentum hold either positive or negative signs. For example, in Case- 1 (non-linear model) the coefficient of $-\sin \phi /(\rho-\tau)^{2}$ is $-6.003 \cdot 10^{3}$ with the rotation 1 , but it changes to $7.215 \cdot 10^{3}$ with the rotation 2 . $-\sin \phi /(\rho-\tau)^{2}$ is one of the projected momenta of $1 /(\rho-\tau)^{2}$ in the direction of $-\sin \phi \cdot \mathrm{W}_{11}$, where $1 /(\rho-\tau)^{2}$ represents the first and the second terms of the equation (39), $16 /\left\{9(\rho-\tau)^{2}\right\}$ and $8 /\left\{3(\rho-\tau)^{2}\right\}$.

Then we compare the changes of the spin angular momentum with the changes of the energy intensity. The energy intensity of $1 /(\rho-\tau)^{2}$ in Table 2 for Case- 1 (non-linear model) changes its sign from negative to positive when the rotation of the object becomes faster from rotation 1 to rotation 2 . It suggests that this component is a negative flow of the waves when the object rotates with the frequency of $\phi_{1}$, but when the object rotates faster with the frequency of $\phi_{2}$, the waves become a positive flow; and, the spin angular momentum also changes its spinning direction from negative to positive as shown in Table 5.

Table 5: Relative intensity of spin angular momentum, Case-1

\begin{tabular}{|l|c|c|}
\hline Components of the waves & Rotation 1 & Rotation 2 \\
\hline$-\sin \phi /(\rho-\tau)^{2}$ & $-6.003 \cdot 10^{3}$ & $7.215 \cdot 10^{3}$ \\
\hline$-\sin \phi /(\rho-\tau)^{11 / 3}$ & $1.400 \cdot 10^{6}$ & $-1.734 \cdot 10^{6}$ \\
\hline$-\sin \phi /(\rho-\tau)^{13 / 3}$ & $4.592 \cdot 10^{6}$ & $-5.688 \cdot 10^{6}$ \\
\hline$-\sin \phi \sin ^{2} \theta$ & $-1.163 \cdot 10^{-2}$ & $-1.234 \cdot 10^{-2}$ \\
\hline $\sin \phi\left(\cos ^{2} \theta-\sin ^{2} \theta\right)$ & 0.2898 & -0.5123 \\
\hline
\end{tabular}

Table 6: Relative intensity of spin angular momentum, Case-2

\begin{tabular}{|l|c|c|}
\hline Components of the waves & Rotation 1 & Rotation 2 \\
\hline$-\sin \phi /(\rho-\tau)^{2}$ & -71.08 & 7.644 \\
\hline$-\sin \phi /(\rho-\tau)^{11 / 3}$ & $5.946 \cdot 10^{2}$ & $1.031 \cdot 10^{2}$ \\
\hline$-\sin \phi /(\rho-\tau)^{13 / 3}$ & $6.159 \cdot 10^{2}$ & $1.605 \cdot 10^{2}$ \\
\hline$-\sin \phi \sin ^{2} \theta$ & $-2.250 \cdot 10^{-4}$ & $-1.270 \cdot 10^{-2}$ \\
\hline $\sin \phi\left(\cos ^{2} \theta-\sin ^{2} \theta\right)$ & 1.129 & -0.5360 \\
\hline
\end{tabular}

The summations of the values for positive and negative coefficients of the spin angular momenta are shown in Table 7 for Case- 1 and Case-2, and Figure 8 for Case- 1 and Figure 9 for Case2 . In both cases, the values change upon the selection of the rotation-mode of the object, rotation 1 or rotation 2. As shown in 
the figures, the spin angular momentum changes its sign between rotation 1 and rotation 2 in Case-1 (non-linear model), while such a rule of changing sign is not so clear in Case-2 (linear model).

Table 7: Relative intensity of spin angular momentum

\begin{tabular}{|l|l|l|}
\hline & Positive flow & Negative flow \\
\hline Case-1 Rotation 1 & $5.992 \cdot 10^{6}$ & $-6.003 \cdot 10^{3}$ \\
\hline Case-1 Rotation 2 & $7.215 \cdot 10^{3}$ & $-7.421 \cdot 10^{6}$ \\
\hline Case-2 Rotation 1 & $1.212 \cdot 10^{3}$ & $-7.108 \cdot 10^{1}$ \\
\hline Case-2 Rotation 2 & $2.713 \cdot 10^{2}$ & $-5.486 \cdot 10^{2}$ \\
\hline
\end{tabular}

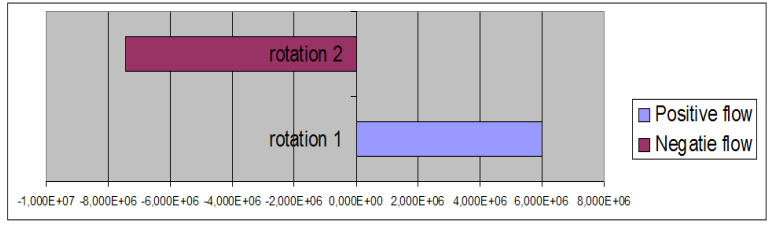

Figure 8: Relative intensity of spin angular momentum Case-1

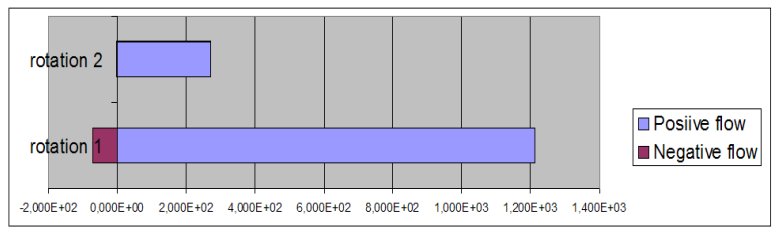

Figure 9: Relative intensity of spin angular momentum Case-2

\subsection{Discussion}

The results show that positive and negative flows of the waves appear if the object rotates, and some of the components that indicate positive and negative flows have opposite spinning directions. The results also show that the rotation-mode (rotation 1 or rotation 2) of the object relates to the energy intensity of the emitted waves, as well as their spinning directions.

Table 8 and Table 9 for Case-1 (non-linear model) and Table 10 and Table 11 for Case-2 (linear model) summarize the changes of the signs of the energy intensity and the spin angular momentum.

In Case-1 (non-linear model), except $\sin ^{2} \theta$, these components change their energy intensities either from positive to negative or from negative to positive, and simultaneously change their signs of the spin angular momenta either from positive to negative or from negative to positive. For example, the component of $1 /(\rho-\tau)^{2}$ is a negative flow with rotation 1 , but it is a positive flow with rotation 2; while its spinning direction changes from negative to positive when the rotation-mode changes from rotation 1 to rotation 2.

The coefficients of $1 /(\rho-\tau)^{11 / 3},-1 /(\rho-\tau)^{13 / 3}$ and $\cos ^{2} \theta-\sin ^{2} \theta$ show the same rule of changing sign, but in the opposite directions of the flow and the spin of $1 /(\rho-\tau)^{2}$.
This finding in Case-1 (non-linear model) is consistent with Figure 10 of our previous research [5] upon the analysis in rectilinear coordinates, in which the negative flow has the clockwise spin, while the positive flow has the anti-clockwise spin.

The exception, $\sin ^{2} \theta$, relates to $\cos \phi \sin ^{2} \theta$ of Table 2 for the energy intensity and to $\sin \phi \sin ^{2} \theta$ of Table 5 for the spin angular momentum. The absolute values of these calculated coefficients are smaller than those of the other components; and, the flow and the spin don't change their signs. Therefore this component is not considered significant.

In Case-2 (linear model) shown in Table 10 and Table 11, the rule of changing sign as observed in Case-1 applies only to $\cos ^{2} \theta-$ $\sin ^{2} \theta$.

Table 8: The sign of the coefficient, energy intensity, Case-1

\begin{tabular}{|c|c|c|c|}
\hline & No rotation & Rotation 1 & Rotation 2 \\
\hline $1 /(\rho-\tau)^{2}$ & + & - & + \\
\hline $1 /(\rho-\tau)^{11 / 3}$ & - & + & - \\
\hline$-1 /(\rho-\tau)^{13 / 3}$ & - & + & - \\
\hline $\sin ^{2} \theta$ & + & + & + \\
\hline $\cos ^{2} \theta-\sin ^{2} \theta$ & + & + & - \\
\hline
\end{tabular}

Note for Tables 8, 9, 10 and 11: The component of $\cot ^{2} \theta$ doesn't appear in these tables because it is on the rotation axis, and not considered. $1 /(\rho-\tau)^{2}$ is a simplified form of $16 / 9(\rho-\tau)^{2}$ and $8 / 3(\rho-\tau)^{2} ;-1 /(\rho-\tau)^{11 / 3}$ is a simplified form of $-64 \mathrm{~m} / 27 \mu(\rho-$ $\tau)^{11 / 3}$; $\sin ^{2} \theta$ is a simplified form of $8 \mu^{3} \sin ^{2} \theta / 18 m$ of the equation (39); and $\cos ^{2} \theta$ $\sin ^{2} \theta$ is a simplified form of $8 \cos ^{2} \theta-8 \sin ^{2} \theta$ of the equation (40). All these components are multiplied by $\cos \phi$ for calculating the energy intensity and by $\sin \phi$ and $\sin \phi$ for calculating the spin angular momentum.

Table 9: The sign of the coefficient, Spin angular momentum, Case-1

\begin{tabular}{|c|c|c|}
\hline & Rotation 1 & Rotation 2 \\
\hline$-\sin \phi /(\rho-\tau)^{2}$ & - & + \\
\hline$-\sin \phi /(\rho-\tau)^{11 / 3}$ & + & - \\
\hline$-\sin \phi /(\rho-\tau)^{13 / 3}$ & + & - \\
\hline$-\sin \phi \sin ^{2} \theta$ & - & - \\
\hline $\sin \phi\left(\cos ^{2} \theta-\sin ^{2} \theta\right)$ & + & - \\
\hline
\end{tabular}

Table 10: The sign of the coefficient, energy intensity, Case-2

\begin{tabular}{|c|c|c|c|}
\hline & No rotation & Rotation 1 & Rotation 2 \\
\hline $1 /(\rho-\tau)^{2}$ & - & - & - \\
\hline
\end{tabular}




\begin{tabular}{|c|c|c|c|}
$1 /(\rho-\tau)^{11 / 3}$ & + & - & + \\
\hline$-1 /(\rho-\tau)^{13 / 3}$ & + & - & + \\
\hline $\sin ^{2} \theta$ & + & + & + \\
\hline $\cos ^{2} \theta-\sin ^{2} \theta$ & + & + & - \\
\hline
\end{tabular}

Table 11: The sign of the coefficient, Spin angular momentum, Case-2

\begin{tabular}{|c|c|c|}
\hline & Rotation 1 & Rotation 2 \\
\hline$-\sin \phi /(\rho-\tau)^{2}$ & - & + \\
\hline$-\sin \phi /(\rho-\tau)^{11 / 3}$ & + & + \\
\hline$-\sin \phi /(\rho-\tau)^{13 / 3}$ & + & + \\
\hline$-\sin \phi \sin ^{2} \theta$ & - & - \\
\hline $\sin \phi\left(\cos ^{2} \theta-\sin ^{2} \theta\right)$ & + & - \\
\hline
\end{tabular}

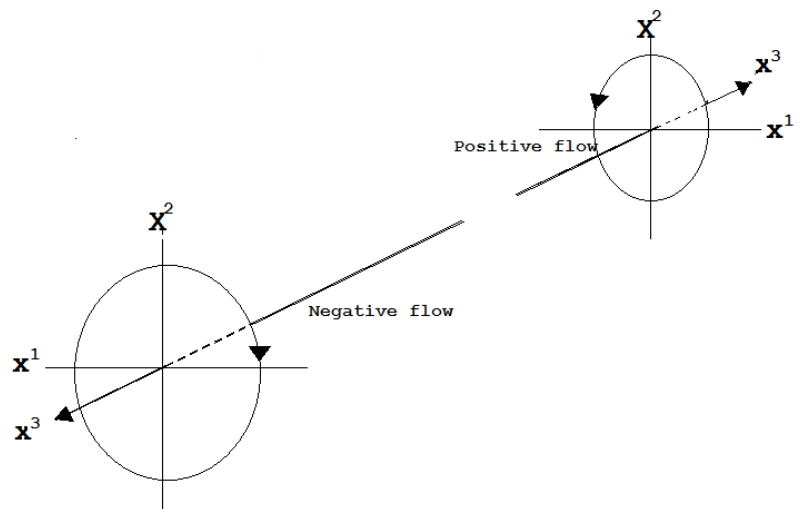

Figure 10: Geometrical relation between positive and negative flows in flat space (from [5])

\section{Conclusion and recommendation}

The result indicates that the rotation of the object can produce both positive and negative energy intensities of the waves, and they are interpreted as positive and negative flows. Both positive and negative flows appear when the object rotates, although the selection of the input data, the non-linear model or the linear model, leads to differences of the energy intensities.

With the non-linear model, the calculated spin angular momentum of the waves changes its sign, + or - , when most of the components change the signs of their energy intensities either from positive to negative or from negative to positive, depending on the speed of the object's rotation. This finding means that most of the components of the waves change their spinning directions when the energy intensity of the flow of the waves changes its sign + or - , also depending on the speed of the object's rotation.
This particular finding on the spin angular momentum is consistent with the previous research [5]. However, with the linear model, most of the components of the waves don't follow the same rule of changing sign.

A parallel image of the source of the anti-gravity is a rotating black hole, and the same rule may apply. Then the positive flow of the waves may be gravitational waves and the negative flow may be the gravitational waves that flow in opposite directions. If those gravitational waves are observed from a rotating black hole and if they indicate the same rule of the energy intensity and the angular momentum as shown in this paper, it will certify the theory.

On the other hand, the theory of the time-space distortion should be verified with a compact nuclear fusion reactor for developing the flying craft. At the next step of the research, it is necessary to calculate the probability of proton-capture or protoncharge-exchange with the time-space distortion model that is used for the simulation reported in this paper.

\section{References}

[1] Y. Matsuki, P.I. Bidyuk, "Theory and Simulation of Artificial Antigravity,” 2020 IEEE 2nd International Conference on System Analysis Intelligent Computing, 2020, doi: 10.1109/SAIC51296.2020.9239195.

[2] Y. Matsuki, P.I. Bidyuk, "Numeric Simulation of Artificial Antigravity upon General Theory of Relativity,” Advances in Science, Technology and Engineering Systems Journal, 6(3), 45-53, 2021, doi: 10.25046/aj060307.

[3] P.A.M. Dirac, General Theory of Relativity, New York: Florida University, A Wiley Inter-science Publication, John Wiley \& Sons, 1975.

[4] H. Goldstein, C.P. Poole, J.L. Safko, Classical Mechanics, 3rd Edition published by Pearson Education. Inc., 2002.

[5] Y. Matsuki, P.I. Bidyuk, "Analysis of Negative Flow of Gravitational Waves," System Research \& Information Technology, N4, 7-18, 2019, doi: 10.20535/SRIT.2308.8893.2019.4.01. 